\title{
SIMPLE ARCHIMEDEAN DIMENSION GROUPS
}

\author{
DAVID HANDELMAN
}

(Communicated by Birge Huisgen-Zimmermann)

\begin{abstract}
We answer affirmatively a question of Goodearl on the existence of simple archimedean dimension groups with arbitrary Choquet simplex as a trace space - at least when the Choquet simplex is metrizable.
\end{abstract}

A partially ordered abelian group $G$ is an abelian group together with a subset denoted $G^{+}$(the positive cone of $G$ ) such that $G^{+}+G^{+} \subseteq G^{+}, G^{+} \cap-G^{+}=\{0\}$, and $G^{+}$generates $G$ as an abelian group. If $g$ and $h$ are elements of $G$, we abbreviate $g-h \in G^{+}$to $h \leq g$. An order unit of the partially ordered abelian group $G$ is an element, $u$, of the positive cone such that for all $g$ in $G$, there exists a positive integer $N$ such that $g \leq N u$.

Let $(G, u)$ be an unperforated partially ordered abelian group with an order unit, $u$. It is simple if every nonzero positive element is an order unit. It is archimedean if for elements $g$ and $h$ of $G, n g \leq h$ for all positive integers $n$ implies $-g \geq 0$; this is the classical definition of archimedean. By a trace (in older and original terminology, as in [G], it is a state), we mean a nonzero positive additive function $\tau: G \rightarrow \mathbf{R}$. A trace, $\tau$, on $G$ is normalized with respect to an order unit $u$ of $G$ if $\tau(u)=1$.

In the point-open topology, the collection of normalized traces forms a compact convex subset of a dual Banach space, denoted $S(G, u)$. A (normalized) trace $\tau$ is extremal (or pure) if whenever $\sigma$ and $\phi$ are normalized traces and there exists $0<\lambda<1$ such that $\tau=\lambda \phi+(1-\lambda) \sigma$, then $\tau=\sigma=\phi$. The set of extreme traces in $S(G, u)$ is denoted by $\partial_{e} S(G, u)$. The vector space consisting of the convexlinear (affine) continuous functions $f: S(G, u) \rightarrow \mathbf{R}$ is denoted by Aff $S(G, u)$. We use $^{\wedge}$ to denote the natural map $G \rightarrow \operatorname{Aff} S(G, u)$, given by $\hat{g}(\tau)=\tau(g)$.

A dimension group is an unperforated partially ordered (abelian) group satisfying the Riesz interpolation property (given $a, b \leq c, d$ in $G$, there exists $e$ in $G$ such that $a, b \leq e \leq c, d)$. For more details and results relating to dimension groups and Choquet theory, see $\mathrm{G}$. (especially sections $3-7,10,11,13,14$ ).

By [G. Theorem 4.14], in the presence of an order unit, the archimedeanness of $G$ is equivalent to the condition that for $g$ in $G, \tau(g) \geq 0$ for all pure traces $\tau$ implies $g \in G^{+}$. When $G$ is archimedean, the natural map $G \rightarrow \operatorname{Aff} S(G, u)$ is an embedding (an order-embedding, in fact). A much weaker definition -irrelevant here-is used by a large group of workers in real algebraic geometry, causing confusion.

Received by the editors March 28, 2011 and, in revised form, January 22, 2012.

2010 Mathematics Subject Classification. Primary 06F20, 46A55.

The author was supported in part by a Discovery Grant from NSERC. 
Among unperforated groups, archimedean and simple represent properties that are maximal and minimal, respectively. Simple groups are those for which a sufficient condition for an element to be positive, namely $\tau(g)>0$ for all traces $\tau$, is necessary as well (for nonzero $g$ ). Archimedean partially ordered abelian groups (with order unit) are those for which the minimal necessary condition on an element to be in the positive cone, $\tau(g) \geq 0$ for all traces $\tau$, is sufficient.

So, it is somewhat difficult to construct dimension groups that are both simple and archimedean, aside from subgroups of the reals. Question 4 of [G. p. 318] asks whether every Choquet simplex can be the trace space of a simple archimedean dimension group; we show this is the case for metrizable simplices (using countable dimension groups), based on an interesting construction over the interval.

Simple dimension groups have been characterized [GH1, Corollary 4.10]; given a countable torsion free abelian group and a group homomorphism $\phi: G \rightarrow \operatorname{Aff} K$ where $K$ is a Choquet simplex and $\phi$ has dense image, define $G^{+} \backslash\{0\}$ to be $\{g \in G \mid \phi(g)$ is strictly positive $\}$. The result is a simple dimension group, and all simple dimension groups other than $\mathbf{Z}$ are of this form. If there exists $u$ in $G$ such that $\phi(u)$ is the constant function with value one, then $K$ may be identified with $S(G, u)$.

An alternative formulation of simple and archimedean groups (in the presence of an order unit) is the following. Suppose $G$ admits an unperforated partial ordering such that the map $G \rightarrow \operatorname{Aff} S(G, u)$ is an embedding; then the ordering is unique (the smallest ordering is strict, the largest ordering is pointwise, as functions on $S(G, u)$ ) with respect to the property that the group be a dimension group with $S(G, u)$ as a trace space.

The following is practically tautological.

Lemma 1. Let $(G, u)$ be an unperforated partially ordered abelian group with order unit. Then $G$ is simple and archimedean if and only if for all $g \in G \backslash\{0\}$, $\inf _{\tau \in \partial_{e} S(G, u)} \tau(g) \neq 0$.

Proof. As a consequence of [G, Corollary 6.4], $\inf _{\tau \in \partial_{e} S(G, u)} \tau(g)>0$ implies $\inf _{\tau \in S(G, u)} \tau(g)>0$ (and thus the infima are equal).

Assume $G$ is archimedean and simple; the former says that $\inf _{\tau \in \partial_{e} S(G, u)} \tau(g)=0$ entails $g \in G^{+}$; simplicity implies $g$ would be an order unit, hence the infimum would be strictly greater than zero.

Conversely, suppose nonzero $g$ satisfies $\tau(g) \geq 0$ for all pure $\tau$. By hypothesis (the infimum is not zero, hence must be strictly positive), $\tau(g)>0$ for all pure $\tau$. Hence (by the first paragraph), $\tau(g)>0$ for all traces, whence $g$ is an order unit and thus in the positive cone, so $G$ is simple and archimedean (simultaneously).

Note that the criterion refers to all nonzero $g$, not just those in $G^{+}$(which would characterize simplicity). In particular, a simple archimedean group which is also an ordered real vector space must be the reals with the usual ordering: pick nonzero $g$ in $G^{+}$, and let $\alpha=\inf \tau(g)$; then $\hat{g}-\alpha \hat{u}$ vanishes at an extreme point (the zero set of $\hat{g}-\alpha \hat{u}$ is a closed face, therefore contains an extreme point) and is nonnegative everywhere; archimedeanness entails $g \geq \alpha u$, and simplicity then forces $g=\alpha u$ (since $g-\alpha u$ is killed by a trace, it cannot be an order unit, so it must be zero).

An extreme version is the following. An unperforated partially ordered abelian group with order unit, $(G, u)$, is extremely simple if for all $g \in G \backslash\{0\}$ and all pure traces $\tau, \tau(g) \neq 0$. 
To see that this implies the criterion of Lemma 1, we use the first remark in the first paragraph of the argument - if $G$ is extremely simple and $\tau(g) \geq 0$ for all pure $\tau$, the hypothesis ensures that $\tau(g)>0$ for all pure $\tau$, and by [G, Corollary 6.4] again, $g$ is an order unit, so the infimum is strictly positive.

Obvious examples are subgroups of the reals with the relative ordering. There are others.

\section{EXAMPLES OF EXTREMELY SIMPLE DIMENSION GROUPS WITH MODESTLY INTERESTING TRACE SPACES}

1. [EHS, [H2]. Let $f$ be a monic integer irreducible polynomial whose roots are all real, $\left\{r_{1}, r_{2}, \ldots, r_{n}\right\}$. Form the ring $R=\mathbf{Z}\left[r_{1}\right]$, and denote by $\tau_{i}: R \rightarrow \mathbf{R}$ the unique multiplicative map obtained by sending $r_{1} \mapsto r_{i}$. Taking only the first $n-1$ of them, we obtain a map $\left(\tau_{1}, \ldots, \tau_{n-1}\right): R \rightarrow \mathbf{R}^{n-1}$, yielding an embedding of $R$ in $\mathbf{R}^{n-1}$. Equip $R$ with the strict ordering, that is, $r \in R^{+} \backslash\{0\}$ if and only if $\tau_{i}(r)>0$ for all $i=1,2, \ldots, n-1$.

This makes $R$ into a simple partially ordered group, and also a partially ordered ring (since the $\tau_{i}$ are ring homomorphisms). The image is dense (as has been computed in [EHS]) so that $G$ is a simple dimension group, free of rank $n$, with 1 as an order unit, and its pure traces are exactly $\left\{\tau_{i}\right\}_{i=1}^{n-1}$. Since each $\tau_{i}$ is one to one, $R$ is extremely simple. In this case, the pure trace space consists of $n-1$ points.

2. Suppose that $K$ is an algebraic extension field of $\mathbf{Q}$, and in addition $K$ is formally real; i.e., if not all $k_{i}$ are zero, then $\sum k_{i}^{2} \neq 0$ (or what amounts to the same thing, $K$ admits a real embedding). Impose on $K$ the sums of squares ordering (that is, $K^{+}$consists of the set of sums of squares). As $K$ is formally real, this is a proper cone, and from algebraicity, it follows and is easy to check that 1 is an order unit for $K$; moreover, inverses of positive elements are positive, and multiplication preserves the positive cone. It is known that $K$ is a dimension group; i.e., it satisfies interpolation (this is true for any formally real field, not just algebraic extensions of the rationals, [H1, 2.11]). For a partially ordered ring with 1 as the order unit, the pure traces are multiplicative; in particular, they are ring homomorphisms [H3, Lemma I.1]. Since $K$ is a field, none of these has a nontrivial kernel, verifying extreme simplicity. In particular, $K$ is a simple archimedean dimension group, which is also an ordered ring.

The pure trace space can be interesting. Let $K$ be $\mathbf{Q}_{\mathbf{R}}$, the subfield of the reals consisting of all elements algebraic over $\mathbf{Q}\left(\mathbf{Q}_{\mathbf{R}}\right.$ is of index two in the algebraic closure of $\mathbf{Q}$ ). Then the pure traces can be identified with the Galois automorphisms of $\mathbf{Q}_{\mathbf{R}}$ and, in particular, the pure trace space is the nonatomic, totally disconnected, separable compact set (sometimes called a Cantor set).

Extreme simplicity is drastic, as evidenced by the trivial Lemma 2.

Lemma 2. Suppose that $(G, u)$ is a dimension group with order unit and there exists a connnected subset $U$ of $\partial_{e} S(G, u)$. Then there exist $g$ in $G$ and $s$ in $U$ such that $\hat{g}$ is not constant on $U$ and $s(g)=0$.

Proof. Given distinct $v$ and $w$ in $U$, there exists $h$ in $G$ such that $\hat{h}(v) \neq \hat{h}(w)$. Since $\hat{h} \mid U$ is continuous and $U$ is connected, the range of $\hat{h} \mid U$ is a nontrivial interval. Let $q=a / b$ be a rational number (with $a$ an integer and $b$ a positive integer) in 
the interval. There exists $s$ in $U$ such that $\hat{h}(s)=q$. Set $g=b h-a u$; since $\hat{h}$ is nonconstant on $U$ and $\hat{u}$ is the constant function $1, \hat{g}$ is not constant on $U$.

In particular, if $(R, 1)$ is an unperforated partially ordered ring with 1 as an order unit, and $R$ is extremely simple, then the pure trace space (known to be compact, since the pure traces are exactly the multiplicative ones) must be totally disconnected, as in example 2 above. In fact, we require only that the ordered ring $R$ be simple and archimedean.

Corollary 3. Suppose $(R, 1)$ is a partially ordered ring with 1 as an order unit, and $R$ is a simple and archimedean dimension group. Then the pure trace space $\partial_{e} S(R, 1)$ is a compact totally disconnected space.

Proof. Compactness follows from [H3, I.1]. If $\partial_{e} S(R, 1)$ contained a connected subset, then by Lemma 2, there would be an element $r$ together with a pure trace $x$ such that $x(r)=0$, and moreover archimedeanness entails $\hat{r} \neq 0$, so $r^{2}$ is not zero. Then $r^{2}$ is nonnegative at every pure trace (since all such are multiplicative); hence by archimedeanness, $r^{2}$ would belong to $R^{+}$, and of course, $x\left(r^{2}\right)=0$, contradicting Lemma 1.

This seems about the end of the road for extremely simple (dimension) groups.

Example 4. Simple archimedean dimension groups with the unit interval as pure trace space.

Proof. Recall from example 2 above the maximal algebraic (over the rationals) subfield of the reals, $\mathbf{Q}_{\mathbf{R}}$. Let $\left\{\alpha_{i}\right\}_{i \in \mathbf{N}}$ be a countably infinite set of real numbers such that the enlarged set $\{1\} \cup\left\{\alpha_{i}\right\}_{i \in \mathbf{N}}$ is linearly independent over $\mathbf{Q}_{\mathbf{R}}$; e.g., if $t$ is a transcendental number, we could take $\alpha_{i}=t^{i}$, or we could simply insist that $\left\{\alpha_{i}\right\}$ be algebraically independent over $\mathbf{Q}$.

Inside the real polynomial algebra $\mathbf{R}[x]$, define the elements, $e_{0}=1, e_{i}=x^{i}-\alpha_{i}$ $(i=1,2,3, \ldots)$, and define $G \subset \mathbf{R}[x]$ to be the rational span of $\left\{e_{j}\right\}_{j \geq 0}$.

Equip $\mathbf{R}[x]$ with the strict ordering as a subgroup of $C([0,1], \mathbf{R})$ so that $\mathbf{R}[x]$ is a simple dimension group (since the image is dense), and put the relative ordering on $G$. Automatically, $G$ is simple. Next, $G$ is a rational vector space, so its closure with respect to the supremum norm - equivalently the norm on $\mathbf{R}[x]$ with the strict ordering - is a real vector space, and thus each $x^{i}$ is contained in the closure of $G$. Hence $G$ is dense in $C([0,1], \mathbf{R})$ (since $\mathbf{R}[x]$ is), and in particular, its pure trace space is the same as that of $\mathbf{R}[x]$, namely $[0,1]$, and moreover, $G$ is a dimension group.

We show that $G$ is archimedean by verifying the condition of Lemma 1 . The pure traces of $G$ are evaluations at points of $[0,1]$; that is, $f \mapsto f(\alpha)$ for $0 \leq \alpha \leq 1$.

Pick (to begin with) an arbitrary nonzero element of $G, g=q_{0}+\sum_{1}^{n}\left(x^{i}-\alpha_{i}\right) q_{i}$, where $q_{i}$ are rationals; since $g$ is not zero, not all the rational coefficients are zero. If $\alpha$ is a real number that is algebraic over the rationals, then $g(\alpha)=0$ entails $q_{0}+\sum_{1}^{n} q_{i}\left(\alpha^{i}-\alpha_{i}\right)=0$, yielding the equation $q_{0}+\sum_{i=1} q_{i} \alpha^{i}=\sum q_{i} \alpha_{i}$; the left side is algebraic (over the rationals), hence belongs to $\mathbf{Q}_{\mathbf{R}}$, while the right side is a rational- (hence a $\mathbf{Q}_{\mathbf{R}^{-}}$) linear combination of $\left\{\alpha_{i}\right\}$. By our assumption, both sides must be zero, which forces $q_{1}=q_{2}=\cdots=0$, and this in turn forces $q_{0}=0$, a contradiction. The conclusion is that if $g$ is an element of $G$ and not a constant, then it cannot have any zeros at algebraic points. 
Now suppose that nonzero $g$ in $G$ has minimum 0 as a function on the unit interval. Then $g(\alpha)=0$ for some $\alpha$ in the unit interval. By the preceding paragraph, $\alpha$ is not algebraic, so in particular, cannot be zero or one; thus it must be an interior point, and since $g$ is nonnegative, $\alpha$ is the location of a minimum of $g$ (as a continuous function on $[0,1])$. Since $g$ is a polynomial and $\alpha$ is an interior point of the interval, we must have $g^{\prime}(\alpha)=0$. But this entails $\sum q_{i} i \alpha^{i-1}=0$, which in turn entails either that $\alpha$ is algebraic or that $q_{1}=q_{2}=\cdots=q_{n}=0$, hence $q_{0}=0$; either way, we reach a contradiction. Thus $g$ in $G \backslash\{0\}$ with $g \mid[0,1] \geq 0$ forces $g$ to have no zeros in $[0,1]$. This verifies the criterion of Lemma 1.

Thus $G$ is a simple archimedean dimension group whose pure trace space is the unit interval.

A sensitivity phenomenon occurs if we change the endpoints of the interval from $[0,1]$ to $[a, b]$. If both $a$ and $b$ are algebraic, then the same argument applies (since the putative functions cannot vanish at either endpoint; hence any zeros must be in the interior, whence the derivative argument works). On the other hand, if either $a$ or $b$ is of the form $\alpha_{k}^{1 / k}$ (where this makes sense, e.g., if $k$ is even, then $\alpha_{k}$ must be positive) for some $k$, then one of $\pm e_{k}= \pm\left(x^{k}-\alpha_{k}\right.$ ) (an element of $G$ ) will vanish at one point of the interval while being strictly positive on the rest of it. In particular, the so-constructed simple dimension group $G$ will not be archimedean.

The idea underlying Example 4 yields a complete answer (at least in the metrizable case) to Goodearl's question.

Example $\infty$. For every metrizable Choquet simplex $K$ there exists a countable simple archimedean dimension group whose trace space is (affinely homeomorphic to) $K$. If the continuum hypothesis is negated, there exist some nonmetrizable Choquet simplices together with simple archimedean dimension groups whose trace space is $K$.

Proof. Let $K$ be an infinite-dimensional Choquet simplex with extremal boundary $\partial_{e} K$, and suppose there is a dense real subspace $L$ of $A:=$ Aff $K$ of infinite dimension (as a real vector space) $\aleph$ strictly less than $2^{\aleph_{0}}$. (This will occur if $K$ is metrizable and not finite-dimensional, so we can take $\aleph=\aleph_{0}$. If the negation of the continuum hypothesis holds, there will exist nonmetrizable such $K$ as well, obviously with $\aleph>\aleph_{0}$.) Without loss of generality, we may assume the constant function 1 belongs to $L$. There exists a basis $\left\{u_{\omega}\right\}_{\omega \in \Omega}$ (over the reals) for $L$, with $0 \in \Omega$, where $u_{0}$ is the constant function 1 . Obviously $|\Omega|=\aleph$.

For $g$ in $A$, define $s_{-}(g)=\inf _{\tau \in \partial_{e} K} g(\tau)$. We note that for any real number $\lambda, s_{-}(g-\mathbf{1} \lambda)=s_{-}(g)-\lambda$. For a subgroup $J$ of $A$, denote by $s(J)$ the subgroup of $\mathbf{R}$ generated by the set of values of $s_{-}(g)$ as $g$ varies over $J$. Obviously $s(J)$ is a subgroup of the reals. If $J$ is a rational vector space, so is $s(J)$, and equally obviously $|s(J)| \leq \aleph_{0} \cdot|J|$. (It is not clear that the set of values $\left\{s_{-}(g)\right\}_{g \in J}$ is itself a group; fortunately, this does not matter, since only the cardinality of the group it generates is relevant.)

Let $H$ be the rational span of $\left\{u_{\omega}\right\}_{\omega \in \Omega}$ (that is, $H$ is the rational vector space with basis $\left.\left\{u_{\omega}\right\}_{\omega \in \Omega}\right)$. In particular, since $|H|=|\Omega|=\aleph$, it follows that $|s(H)| \leq \aleph$. By hypothesis, this is strictly less than $2^{\aleph_{0}}=|\mathbf{R}|$. Hence the dimension (over $\mathbf{Q}$ ) of the quotient vector space $\mathbf{R} / s(H)$ is $2^{\aleph_{0}}$. It follows that we may find a collection $\left\{\lambda_{\omega}\right\}_{\omega \in \Omega \backslash\{0\}}$ of real numbers indexed by $\Omega \backslash\{0\}$ whose image in the quotient is linearly independent (over the rationals, of course). 
Set $v_{0}=u_{0}$, and for all other $\omega$ in $\Omega$, set $v_{\omega}:=u_{\omega}-1 \lambda_{\omega}$. Let $G$ be the rational span of $\left\{v_{\omega}\right\}_{\omega \in \Omega}$. Since $G$ is a rational vector space, its closure in $A$ is a real vector space, and therefore contains each $u_{\omega}$ (as $\mathbf{1}=v_{0}$ belongs to $G$ ), hence contains the dense subspace $L$, so $G$ is dense in $A$. Impose the strict ordering on $G$; as $K$ is a Choquet simplex, $G$ is a simple dimension group whose trace space (normalized with respect to the order unit 1 ) is $K$.

Now we verify the criterion of Lemma 1 for $G$. Pick nonzero $g$ in $G$; if $g$ is a multiple of the constant function, obviously the criterion is satisfied. Otherwise, we may write $g=1 q_{0}+\sum_{W} v_{\omega} q_{\omega}$, where $W$ is a finite subset of $\Omega \backslash\{0\}, q_{0}$ is a rational number, and for all $\omega$ in $W, q_{\omega}$ is a nonzero rational number. Expanding $v_{\omega}=u_{\omega}-\mathbf{1} \lambda_{\omega}$ for all $\omega$ in $W$, we obtain $g=\sum_{W} u_{\omega} q_{\omega}+\mathbf{1}\left(q_{0}-\sum_{W} q_{\omega} \lambda_{\omega}\right)$. Setting $h=\sum_{W} u_{\omega} q_{\omega}+1 q_{0}$, it follows that $h$ belongs to $H$ and $g=h-1 \sum_{W} q_{\omega} \lambda_{\omega}$. Thus $s_{-}(g)=s_{-}(h)-\sum_{W} q_{\omega} \lambda_{\omega}$. If this were zero, it would mean that $\sum_{W} q_{\omega} \lambda_{\omega}$ belongs to $s(H)$, forcing all the $q_{\omega}$ to be zero, a contradiction.

This verifies the criterion of Lemma 1 , so $G$ is a simple archimedean dimension group, a dense subgroup of Aff $K$, and with trace space $K$.

We have excluded finite-dimensional simplices from this construction (simply because the $\aleph$ s and their arithmetic become finite, necessitating prolix explanations), but of course the same method will work, this time with finite and countable sets. Alternatively, we already have examples, even of extremely simple dimension groups (examples 1 and 2) for all finite-dimensional simplices.

\section{REFERENCES}

[EHS] Edward G Effros, David E Handelman, and Chao-Liang Shen, Dimension Groups and Their Affine Representations, American Journal of Mathematics 102 (1980) 385-407. MR.0564479 (83g:46061)

[G] K Goodearl, Partially ordered abelian groups with interpolation, Mathematical Surveys and Monographs \#20, American Mathematical Society (1986). MR0845783 (88f:06013)

[GH1] K Goodearl and D Handelman, Metric completions of partially ordered abelian groups, Indiana Univ Math J 29 (1980) 861-895. MR0589651 (82b:06020)

[GH2] K Goodearl and D Handelman, Tensor products of dimension groups and $\mathrm{K}_{0}$ of unit-regular rings, Canad J Math 38 (1986) 633-658. MR.0845669 (87i:16043)

[H1] D Handelman, Rings with involution as partially ordered abelian groups, Rocky Mountain J Math 11 (1981) 337-381. MR0722571 (84m:16005)

[H2] D Handelman, Free rank $n+1$ dense subgroups of $\mathbf{R}^{n}$ and their endomorphisms, J Funct Anal 46 (1982) 1-27. MR0654462 (84h:06019)

[H3] D Handelman, Positive polynomials and product type actions of compact groups, Memoirs Amer Math Soc 54 (1985) 320. MR0783217(86h:46091)

Department of Mathematics, University of Ottawa, Ottawa ON K1N 6N5, Canada

E-mail address: dehsg@uottawa.ca 